Article

\title{
Application of GISAXS in the Investigation of Three-Dimensional Lattices of Nanostructures
}

\author{
Lovro Basioli ${ }^{1}$, , Krešimir Salamon ${ }^{1}$, Marija Tkalčević ${ }^{1}$, Igor Mekterović ${ }^{2}$, Sigrid Bernstorff ${ }^{3}$ (D) \\ and Maja Mičetić 1 ,*iD \\ 1 Ruđer Bošković Institute, Bijenička cesta 54, Zagreb 10000, Croatia \\ 2 Faculty of Electrical Engineering and Computing, Unska 3, Zagreb 10000, Croatia \\ 3 Elettra-Sincrotrone Trieste S.C.p.A., Strada Statale 14 km 163,500 in AREA Science Park, \\ Basovizza/Trieste 34149, Italy \\ * Correspondence: maja.micetic@irb.hr
}

Received: 22 July 2019; Accepted: 10 September 2019; Published: 13 September 2019

check for updates

\begin{abstract}
The application of the grazing-incidence small-angle X-ray scattering (GISAXS) technique for the investigation of three-dimensional lattices of nanostructures is demonstrated. A successful analysis of three-dimensionally ordered nanostructures requires applying a suitable model for the description of the nanostructure ordering. Otherwise, it is possible to get a good agreement between the experimental and the simulated data, but the parameters obtained by fitting may be completely incorrect. In this paper, we theoretically examine systems having different types of nanostructure ordering, and we show how the choice of the correct model for the description of ordering influences the analysis results. Several theoretical models are compared in order to show how to use GISAXS in the investigation of self-assembled arrays of nanoparticles, and also in arrays of nanostructures obtained by ion-beam treatment of thin films or surfaces. All models are supported by experimental data, and the possibilities and limitations of GISAXS for the determination of material structure are discussed.
\end{abstract}

Keywords: GISAXS; quantum dot (QD) lattices; structure determination

\section{Introduction}

Grazing-incidence small-angle X-ray scattering (GISAXS) is a powerful method for fast and accurate structural analysis of thin-film materials containing nano-objects (NOs), like quantum dots, nanoparticles, nanowires, and similar nanostructures. It is essentially a diffraction method performed at grazing incidence angle to achieve relevant statistics when probing thin films, measured at small diffraction angles to capture objects of a few nanometers in size. The method is especially efficient for the analysis of systems that have some regularity in the NO ordering. Due to the regularity, distinctive Bragg peaks appear in GISAXS maps, because GISAXS reflects the structural properties in the reciprocal space [1-12]. Thus, a visual inspection of a GISAXS map is usually enough to roughly determine the type of the NO arrangement and the separations between the NOs. Of course, a detailed analysis and fitting of a GISAXS map is needed to get the precise values of the structural parameters including NO shape, size, arrangement properties, and their statistical distributions. Several excellent papers describe the GISAXS technique and the general theory behind it [1-3,13]. In addition, there are several very useful software platforms for the analysis of GISAXS data [14-18].

The main problem in the accurate analysis of GISAXS data is that each material type needs a specific model that describes the type of NO ordering inside it [3]. If the model is not properly chosen, it is possible to get good agreement between measured and simulated GISAXS maps, but with wrong values for the structural parameters. However, in some cases, the experimental data cannot be fitted by 
an incorrect model. Additionally, it is desired that the models are simple to use, i.e., to have analytical forms that include statistical distributions. The fitting process is much faster in that case.

In this paper, we present the main models for simulation of GISAXS maps in three-dimensional (3D) lattices of NOs. The models are simple, they contain statistical distributions of all important parameters, and they contain no numerical integrations. We also explain the main differences between different ordering types of NOs and how to distinguish them. We also consider some important problems that can appear during the analysis of GISAXS data, which can easily lead to the wrong interpretation of GISAXS measurements. We concentrate on systems consisting of three-dimensionally (3D)-ordered NOs in thin films, i.e., 3D lattices of NOs, as we have excellent experience in GISAXS analysis of these systems. Such materials are usually produced by self-assembled growth or by ion-beam-assisted deposition [2-12]. Thus, the paper will be especially useful for non-specialists in this field. In addition, we shortly present a new user-friendly software package for modeling GISAXS, suitable for analysis and fitting of all mentioned and similar systems, called GisaxStudio [19]. The models presented here are successfully applied to many systems (some of them in References [2-12]), and they give excellent agreement with the results of microscopy and other structural techniques.

\section{Materials and Methods}

Three types of materials, i.e., 3D lattices of NOs, were considered in this paper: lattices of Ge quantum dots (QDs), networks of Ge nanowires (NWs), and lattices of core/shell Ge/Si QDs. All of these NOs were embedded in an amorphous alumina $\left(\mathrm{Al}_{2} \mathrm{O}_{3}\right)$ matrix. These materials were deposited as thin films by the magnetron sputtering deposition system K.J. Lesker Company (Jefferson Hills, PA, USA), CMS-18, available in the Thin films laboratory of the Ruđer Bošković Institute (Zagreb, Croatia), on $\mathrm{Si}(100)$ substrates. The base pressure was in the range of $10^{-6}-10^{-5} \mathrm{~Pa}$, while the $\mathrm{Ar}$ pressure was $0.66 \mathrm{~Pa}$ in a continuous flow $\left(13 \mathrm{~cm}^{3} \cdot \mathrm{min}^{-1}\right)$. The substrate was rotated (with $1 \mathrm{rpm}$ ) during the depositions to ensure a homogeneous thickness of the layers. The 3D ordering of the NOs was achieved by self-assembled growth type, using surface morphology effects during their nucleation (see References $[8,10,11])$. The sputtering power of the alumina target was $150 \mathrm{~W}$ for all depositions. All depositions were controlled by the software of the KJLC CMS-18 system, ensuring the same deposition conditions for each layer.

The first material type, Ge QDs (denoted by QD1), was deposited by $\left(\mathrm{Ge} / \mathrm{Al}_{2} \mathrm{O}_{3}\right) \times 20$ multilayer deposition at $400{ }^{\circ} \mathrm{C}$ with an addition of nitrogen (6 at.\%). Pure $\mathrm{Ge}$ and $\mathrm{Al}_{2} \mathrm{O}_{3}(99.995 \%)$, produced by K.J. Lesker Company were used as targets in Direct Current (DC) (9 W) and Radio Frequency (RF) $(150 \mathrm{~W})$ magnetron discharge modes, respectively, with a duration of $45 \mathrm{~s}$ for Ge and $110 \mathrm{~s}$ for alumina in each layer. The second type, Ge NWs (denoted by NW1 and NW2), were prepared by continuous $\mathrm{Ge}+\mathrm{Al}_{2} \mathrm{O}_{3}$ co-deposition with duration of $30 \mathrm{~min}$, at $400{ }^{\circ} \mathrm{C}$ for $\mathrm{NW} 1$ and $200{ }^{\circ} \mathrm{C}$ for NW2, while the sputtering powers for the Ge target were $2.5 \mathrm{~W}$ and $10.0 \mathrm{~W}$ for the films NW1 and NW2, respectively. The third material type considered (GeSi1), consisting of Ge/Si core/shell QDs, was prepared by deposition of $\left(\mathrm{Ge} / \mathrm{Si} / \mathrm{Al}_{2} \mathrm{O}_{3}\right) \times 20$ multilayer at $300^{\circ} \mathrm{C}$. Pure $\mathrm{Ge}, \mathrm{Si}$, and $\mathrm{Al}_{2} \mathrm{O}_{3}(99.995 \%)$ were used as targets in DC $(9 \mathrm{~W}), \mathrm{DC}(50 \mathrm{~W})$, and RF $(150 \mathrm{~W})$ magnetron discharge modes, with a duration of 50, 60, and $120 \mathrm{~s}$ respectively. Details of the preparation can be found in References [10,11].

GISAXS measurements were performed at the synchrotron Elettra, Trieste, Italy, at the SAXS beamline, using a photon energy of $8 \mathrm{keV}$, and a two-dimensional (2D) photon detector (Pilatus3 1M). The GISAXS intensity maps were measured using a grazing incidence angle slightly above the critical angle of total reflection.

\section{Results}

\subsection{General Properties of GISAXS Intensity Maps}

In this section, the main features are analyzed that usually appear in a GISAXS map measured on thin films containing NOs. The GISAXS intensity distribution is determined mainly by the shape of 
the NOs (this contribution is called shape factor), and by the arrangement of the NOs (called structure factor). The shape factor is the Fourier transform of the NO shape; thus, it is usually not difficult to define it. However, the structure factor depends on the NO ordering and different approximations have to be applied to get the function that properly describes the NO ordering. Therefore, we firstly concentrate on the description of the structure factor.

A GISAXS intensity distribution in general is given by the following expression [3]:

$$
I(\boldsymbol{Q})=A|\delta \rho|^{2}\left|t_{i} t_{f}\right|^{2}\left\langle\sum_{\boldsymbol{R} \boldsymbol{R}^{\prime}} \Omega_{\boldsymbol{R}}(\boldsymbol{q}) \Omega_{R^{\prime}}^{*}(\boldsymbol{q}) \exp \left[-i\left(\boldsymbol{q} \boldsymbol{R}-\boldsymbol{q}^{*} \boldsymbol{R}^{\prime}\right)\right]\right\rangle,
$$

where $A$ is a constant, $\delta \rho$ is the difference in the electron densities of the NO material and the matrix, $\boldsymbol{R}$ and $\boldsymbol{R}^{\prime}$ are the position vectors of the NOs, $Q$ is the scattering vector, $\boldsymbol{q}$ is the complex scattering vector corrected to refraction at the vacuum-substrate interface, and $\Omega_{R}(q)$ is the shape factor of a NO occurring atposition $\boldsymbol{R}$; the shape factor is derived from the function that is unity in the NO volume and zero outside $i t ; t_{i}$ and $t_{f}$ are the Fresnel transmittivities [20] of the substrate surface corresponding to the primary and scattered waves, respectively $\left(\left|t_{i} t_{f}\right|^{2}\right.$ gives the Yoneda wing in a GISAXS map). The brackets \langle\rangle indicate averaging over the positions and shapes of the NOs. In this paper, we consider the case in which the NO sizes are not correlated with their positions (decoupling approximation); for details about different approximations, please see Reference [1].

As visible from Equation (1), a GISAXS map reflects the properties of the NO shape and ordering in the reciprocal $(Q)$ space. The key parameter for the successful application of Equation (1) is to make good models for the NO shape (shape factor) and NO ordering (structure factor), as mentioned previously. The shape factor $\Omega(q)$ is the Fourier transform of the NO shape; therefore, if we know the shape precisely, it is usually not difficult to calculate it. However, for the determination of the structure factor, the summation should be performed over all pairs of the NO positions $R$ and $\boldsymbol{R}^{\prime}$ within the probed volume. This is the most important task in the determination of the structure factor, and, for that purpose, we must use approximations for the definitions of the NO positions, which make the calculation of the sum and its averaging in Equation (1) easier.

In this paper, we use a paracrystal model to describe the NO positions [3,21]. The paracrystal model for a one-dimensional (1D) case assumes that the position of the $n$-th $\mathrm{NO}$ (at the position $\boldsymbol{R}_{n}$ ) can be expressed as a sum of basis vector $\boldsymbol{a}$ of an ideal (undisturbed) lattice and deviation vector: $\boldsymbol{R}_{n}=n \boldsymbol{a}+\boldsymbol{D}_{n}$, where $\boldsymbol{D}_{n}$ denotes the total deviation of a NO with index $n$ from its ideal position $(n \boldsymbol{a})$. The description of $\boldsymbol{D}_{n}$ depends on the type of NO ordering, as described in detail in the next section.

Sometimes, extra attention should be given to the choice of the shape factor because it may be different than the assumed one. For example, some NOs make a chemical reaction with the surrounding material; thus, a kind of a shell appears around the $\mathrm{NO}$ and we have a different type of shape factor (core/shell instead of the NO alone). We consider the influence of the proper choice of the shape factor in Section 4.3.

\subsection{Ordering Types in Nanostructured Materials}

As explained in the text above using a 1D example $\left(\boldsymbol{R}_{n}\right)$, the positions of the 3D regularly ordered NOs deviate from the ideal positions that they would have in a perfect crystal lattice defined by basis vectors $\boldsymbol{a}_{1}-\boldsymbol{a}_{3}$. While the ideal positions define the position of peaks in the GISAXS map of a certain nanomaterial, the deviations are very important because they are closely related to the intensity distribution of each peak in the map. Therefore, the statistical properties of the NO deviations from the ideal positions (vectors $\boldsymbol{D}_{n}$ ) are crucial for the successful analysis of a GISAXS map. The mathematical description of the statistical properties of the deviations is sometimes complicated. As mentioned before, we use a 3D paracrystal model to describe the NO positions and their deviations. This model treats each component of the basis vectors $\left(\boldsymbol{a}_{1}-\boldsymbol{a}_{3}\right)$ and the related deviations $\left(\boldsymbol{D}_{n}{ }^{1}-\boldsymbol{D}_{n}{ }^{3}\right)$ as independent variables. 
While the ideal positions are precisely defined with the basis vectors, the deviations are usually described by long-range ordering (LRO) or short-range ordering (SRO) models [3]. Additionally, we consider the possibility of a complete absence of regularity in ordering, which leads to the random ordering (RO) model. Figure 1 shows the main differences in these ordering types. We consider a 1D case to keep simplicity, but each component of a basis vector shows the same behavior. Thus, in random ordering, there is no correlation in the NO positions; thus, the structure factor for this type of ordering is a constant. On the other hand, the LRO and SRO assume the existence of correlation in the QD positions (regularity of the NO ordering) with the main difference that the NO positions fluctuate around a predefined separation in the SRO model, while they fluctuate around predefined positions in the LRO model. Therefore, if we fix the position of the first NO (indicated by number 1 in Figure $1 \mathrm{~b}$, the possible positions of the second NO have a distribution characterized by the width parameter $\sigma$ around the ideal position (separation $a$ ). We assume it is a normal distribution with the standard deviation $\sigma$. The possible positions of the third NO in the SRO fluctuate around the ideal position (2a) but with the distribution having a deviation of $2 \sigma$ (see Figure $1 b$ ). Actually, the separations between neighboring NOs fluctuate with the distribution described by $\sigma$, but the width of the distribution describing the position of each $\mathrm{NO}$ from the first $\mathrm{NO}$ (origin) consequently increases. The position of NO in such a $1 D$ SRO model is given by

$$
\boldsymbol{R}_{n}^{S R O}=n \boldsymbol{a}+\sum_{j=1}^{n} \boldsymbol{\delta}_{i} ; \boldsymbol{D}_{n}=\sum_{j=1}^{n} \boldsymbol{\delta}_{i} .
$$
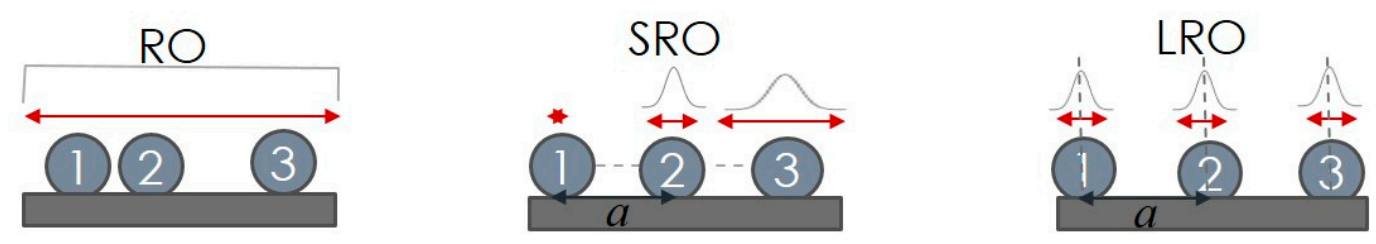

Figure 1. Overview of different models for the description of nano-object (NO) ordering in one dimension (1D). The NOs are indicated by the numbers 1-3. The red arrows and gray lines above them illustrate the probability of a NO position.

The main properties of the LRO model are illustrated in Figure 1c. In this model, the positions are predefined; thus, there is no accumulation of disorder with the distance from the origin. Thus, the position of the second NO can fluctuate around the predefined position $2 a$ with the distribution of width $\sigma$, and it does not depend on the position of any other NO. The position of the $n$-th NO for such a distribution is given by

$$
\boldsymbol{R}_{n}^{L R O}=n \boldsymbol{a}+\boldsymbol{\delta}_{n} ; \boldsymbol{D}_{n}=\boldsymbol{\delta}_{n}
$$

In the 3D case, we have basis vectors $\boldsymbol{a}_{1}-\boldsymbol{a}_{3}$ and we assume that the $x, y, z$ components $\left(\delta_{x ; y ; z}\right)$ of the random deviation $\delta_{n}$ are normally distributed with zero mean and root-mean-square (r.m.s.) dispersion $\sigma_{x}, \sigma_{y}$, and $\sigma_{z}$, respectively. Hence, to describe the system of NOs, we need to know the component of the basis vectors that characterize their ordering $a_{x, y, z}^{1-3}$ (we interchanged the upper and lower indices of the standard notation $a_{1}-a_{3}$ to include the space $x, y, z$ components and to have the same notation as in Reference [3]), number of periods in each space direction $N_{1}-N_{3}$, the disorder parameters in a particular space direction $\sigma_{x, y, z}^{1-3}$, and, of course, the NO shape parameters.

After insertion of Equations (2) or (3) into Equation (1), treating each component independently, the calculation of the sums and averaging is easy to perform analytically; thus, we come to the final equation for the GISAXS intensity distribution in the 3D case as follows:

$$
I(\boldsymbol{Q})=A|\delta \rho|^{2}\left|t_{i} t_{f}\right|^{2} F\left(q_{z}\right)\left\{\left[f_{1}(\boldsymbol{q})-f_{2}(\boldsymbol{q})\right] N_{1} N_{2} \Gamma\left(q_{z}\right)+f_{2}(\boldsymbol{q}) G_{3 D}(\boldsymbol{q})\right\}
$$


where $f_{1}$ and $f_{2}$ are the components of the shape factor, calculated as follows:

$$
f_{1}(\boldsymbol{q})=\left\langle\left|\Omega^{F T}(\boldsymbol{q})\right|^{2}\right\rangle ; f_{2}(\boldsymbol{q})=\left|\left\langle\Omega^{F T}(\boldsymbol{q})\right\rangle\right|^{2},
$$

where $\Omega^{\mathrm{FT}}(\boldsymbol{q})$ is the Fourier transformation of the shape function $\Omega_{R}(\boldsymbol{q})$.

$F\left(q_{z}\right)$ and $\Gamma\left(q_{z}\right)$ are defined as follows:

$$
F\left(q_{z}\right)=\exp \left[-2 \operatorname{Im}\left(q_{z}\right) N_{3} a_{z}^{(3)}\right] ; \Gamma\left(q_{z}\right)=\frac{1-\exp \left[2 \operatorname{Im}\left(q_{z}\right) N_{3} a_{z}^{(3)}\right]}{1-\exp \left[2 \operatorname{Im}\left(q_{z}\right) a_{z}^{(3)}\right]}
$$

where $N_{1}-N_{3}$ are the numbers of the QD position $\left(\boldsymbol{R}_{n 1, n 2, n 3}\right)$ indices (number of periods) in the $x, y$ and $z$ directions, respectively.

$G_{3 D}(q)$ is a product of three $1 D$ correlation functions $G_{o t}^{(i)}(q) ; i=1,2,3$ and they depend on the ordering type in the particular space direction.

$$
\begin{gathered}
G_{S R O}^{(1,2)}(\boldsymbol{q})=N_{1,2}+2 \operatorname{Re}\left\{\frac{\zeta^{(1,2)} \eta^{(1,2)}}{1-\zeta^{(1,2)} \eta^{(1,2)}}\left[N_{1,2}-\frac{\left(\zeta^{(1,2)} \eta^{(1,2)}\right)^{N_{1,2}}-1}{\zeta^{(1,2)} \eta^{(1,2)}-1}\right]\right\} . \\
G_{S R O}^{(3)}(\boldsymbol{q})=\Gamma\left(q_{z}\right)+2 \operatorname{Re}\left\{\frac{\eta^{(3)}}{\zeta^{(3) *}-\eta^{(3)}}\left[\Gamma-\frac{\left(\zeta^{(3)} \eta^{(3)}\right)^{N_{3}}-1}{\zeta^{(3)} \eta^{(3)}-1}\right]\right\} \\
G_{L R O}^{(1,2)}(\boldsymbol{q})=N_{1,2}+\left|\eta^{(1,2)}\right|^{2} 2 \operatorname{Re}\left\{\frac{\zeta^{(1,2)}}{1-\zeta^{(1,2)}}\left[N_{1,2}-\frac{\left[\zeta^{(1,2)}\right]^{N_{1,2}}-1}{\zeta^{(1,2)}-1}\right]\right\} . \\
G_{L R O}^{(3)}(\boldsymbol{q})=\Gamma\left(q_{z}\right)+\left|\eta^{(3)}\right|^{2} 2 \operatorname{Re}\left\{\frac{1}{\zeta^{(3) *}-1}\left[\Gamma-\frac{\left[\zeta^{(3)}\right]^{N_{3}}-1}{\zeta^{(3)}-1}\right]\right\} . \\
G_{M I X}^{(3)}(\boldsymbol{q})=\Gamma\left(q_{z}\right)+\left|\eta_{z}^{(3)}\right|^{2} 2 \operatorname{Re}\left\{\frac{\eta_{\|}^{(3)}}{\zeta^{(3) *}-\eta_{\|}^{(3)}}\left[\Gamma-\frac{\left[\zeta^{(3)} \eta_{\|}^{(3)}\right]^{N_{3}}-1}{\zeta^{(3)} \eta_{\|}^{(3)}-1}\right]\right\} . \\
\zeta^{(j)}(\boldsymbol{q})=\exp \left[-i \boldsymbol{q} \boldsymbol{a}_{j}\right] . \\
\eta_{x, y, z}^{(i)}\left(q_{x, y, z}\right)=\exp \left[-\left(\sigma_{x, y, z}^{i} q_{x, y, z}\right)^{2} / 2\right] ; \eta^{(i)}(\boldsymbol{q})=\eta_{x}^{(i)} \eta_{y}^{(i)} \eta_{z}^{(i)} ; \eta_{\|}^{(i)}=\eta_{x}^{(i)} \eta_{y}^{(i)} ; i=1,2,3 . \\
G_{R O}^{(1,2,3)}(\boldsymbol{q})=\operatorname{const.}
\end{gathered}
$$

We assume that the basis vectors $a_{1}$ and $\boldsymbol{a}_{2}$ are in the $x, y$ plane, while the third basis vector may have all three components, depending on the ordering type. Therefore, only the equations related to the basis vector $\boldsymbol{a}_{3}\left(G_{o t}^{(3)}(\boldsymbol{q})\right.$;) have the refraction effects included (the others contain only the $x$ and $y$ components; thus, there is no need).

Thus, for the successful analysis of a GISAXS map from a particular sample we have to construct the function $G_{3 D}$ (Equation (14)) using 1D functions $G(q)$ (Equations (7)-(13)). We must be careful to choose the correct functions for the description, because a GISAXS map strongly depends on the ordering type. Actually, we must know the main ordering properties of the NOs before starting the GISAXS analysis. These properties usually can be determined from the production process of 
the nanomaterial. If the material is produced by some kind of self-assembly process, then usually the separations fluctuate; therefore, we have to use the SRO model. However, if the positions are predefined from some reason (pre-pattering of the substrate, predefined layer thicknesses (vertical positions of NOs), ion-beam-induced nucleation, ion tracks, etc.), the LRO model should be applied. In some cases, we have a combination of LRO and SRO related to one basis vector. Most usually, this appears in films composed on multilayers with predefined thicknesses, i.e., vertical positions, that contain NOs. If these NOs are ordered by some self-assembly process, then their lateral ( $x$ and $y$ ) components show SRO, while the $z$ component has LRO. In that case, we use Equation (11), i.e., $G_{M I X}^{(3)}(\boldsymbol{q})$, for the description of ordering along basis vector $\boldsymbol{a}_{3}$. We sometimes have to combine several models in different special directions, as illustrated in the examples given later.

We can estimate the ordering type from the measured GISAXS map, if it shows more than one order of peaks related to the NO ordering. In the $Q$-space, the SRO and LRO differ by the behavior of the correlation peaks: the width of the peaks increases with the order of the peak for the SRO, while the peak width is constant for the LRO model and its intensity decreases with the order of the peak (for details, please see the Reference [3]). Hence, the peaks in the GISAXS map show the same basic behavior as the distribution of the NO positions when measured from the origin (Equations (2) and (3)). In Section 4, we examine three examples to illustrate the effects of ordering of NOs and their shape.

\section{Examples}

\subsection{D Ge Quantum Dot Lattices}

In this first example, we consider a 3D lattice of spheroidal NOs (in this example, quantum dots (QDs)) prepared by multilayer deposition. We firstly consider the production process of that material. The material is prepared by computer-controlled deposition of a multilayer (in this example, alternating layers of $\mathrm{Ge}$ and $\mathrm{Al}_{2} \mathrm{O}_{3}$ ); thus, the $z$ position of each layer should be predefined (assuming a constant deposition speed). However, the QDs in this material form by diffusion-mediated nucleation, and their positions within the layers ( $x, y$ plane) are defined by a self-assembly process (see Reference [4]). Therefore, for this particular case, we should use a combination of LRO in the $z$ direction and SRO in the $x$ and $y$ directions. The correct correlation function is

$$
\mathrm{G}_{3 \mathrm{D}}(\boldsymbol{q})=\mathrm{G}_{\mathrm{SRO}}^{(1)}(\boldsymbol{q}) \mathrm{G}_{\mathrm{SRO}}^{(2)}(\boldsymbol{q}) \mathrm{G}_{\mathrm{MIX}}^{(3)}(\boldsymbol{q}) .
$$

The ordering type of QDs for this case is body-centered tetragonal (BCT), which can be concluded from the arrangement and shape of spots and also from the literature data about similar samples [10-12].

The measured GISAXS map from this material and its simulation are shown in Figure 2a,b. From the visual inspection of the measured map, we see only one lateral peak in the $Q_{y}$ direction and two peaks in the $Q_{z}$ direction. Here, we must be careful with the conclusion that there is no more than one peak in the $Q_{y}$ direction, and that the ordering is worse in that direction, because the intensity visible in GISAXS maps also depends on the shape factor. Where the shape factor is zero, no intensity will be visible in the GISAXS map; thus, the shape factor can "make" higher orders not visible.

The second important step in a GISAXS analysis is the inspection of the peak profiles. They are shown in Figure $2 \mathrm{c}, \mathrm{d}$. The profiles are taken along the lines shown in the inset of Figure $2 \mathrm{~d}$. They demonstrate the width of the profiles in the $z$ direction. For both cases, the width of both peaks is nearly the same, showing that the chosen model was correct.

Therefore, when we are sure to have the suitable model of ordering, we can continue with the analysis of the intensity map. The third important step is to determine the lattice type of the NO ordering. This is sometimes difficult, and a complemental microscopy analysis should be applied to be sure that the ordering type is correct, because different ordering types can produce very similar GISAXS maps when they are averaged over azimuthal orientations (due to the appearance of random domains). Another possibility is to have some pre-knowledge about the nucleation properties and the 
production process of the nanomaterial and to make detailed simulations of different structures, to see the differences. One good example is given in Reference [6].
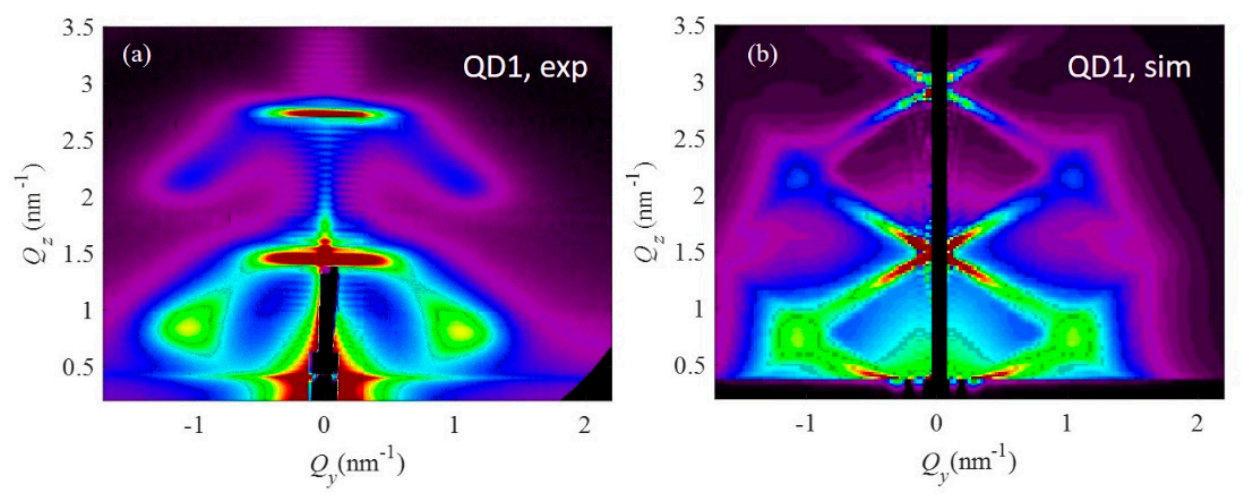

(c)

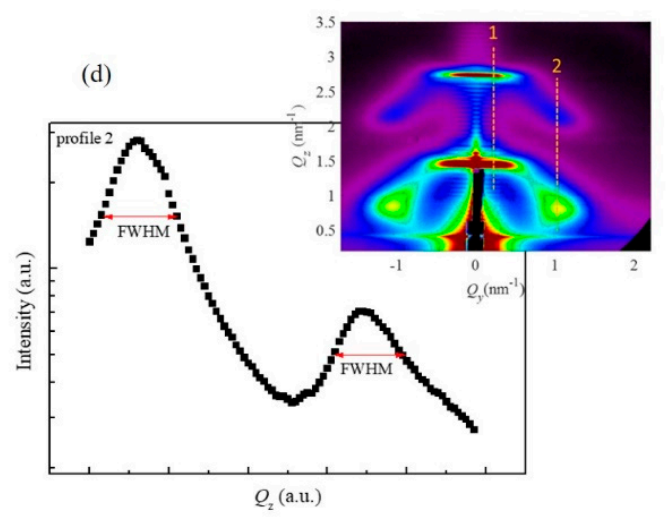

Figure 2. (a) Experimental and (b) simulated grazing-incidence small-angle $X$-ray scattering (GISAXS) map of Ge quantum dots (QDs) embedded in alumina matrix. (c,d) Intensity profiles taken along the lines 1 and 2, respectively, indicated in the inset of (d).

In this particular case, the ordering is body-centered tetragonal (BCT), as illustrated in Figure 3. Thus, for the description of an ideal lattice, we use the basis vectors $\boldsymbol{a}_{1}-a_{3} ; \boldsymbol{a}_{1}$ and $\boldsymbol{a}_{2}$ are placed in the $x$, $y$ plane and have the same length $\left|\boldsymbol{a}_{1}\right|=\left|\boldsymbol{a}_{2}\right|=a$, while the vertical stacking of the QDs is defined by the vector $\boldsymbol{a}_{3}$ (see Figure 3a). As explained before, the multilayer has a predefined position of the single layers; thus, the $z$ components of the QD positions $\left(a_{3} z ;\left|a_{3}{ }^{z}\right|=c\right)$ have LRO, as illustrated in Figure $3 b$. The QDs within the layers show SRO (see Figure $3 c$ ), as they grow by a self-assembly process. It is also important to note that the regular ordering appears in domains randomly rotated around the $z$ axis; thus, azimuthal averaging must be taken into account. The parameters of the analyzed QD lattice obtained by fitting of the experimental map from Figure 2a are given in Table 1, and the simulation is shown in Figure $2 \mathrm{~b}$. The shape of the QDs is assumed to be spheroidal with the lateral and vertical radii $R_{x, y}$ and $R_{z}$, respectively, with the distribution having the standard deviation $\sigma_{R}$. We chose a gamma size distribution because it is very easy to get the analytical expressions for the averaged sizes using it.

Table 1. Parameters of the simulation shown in Figure 2b. QD-quantum dot.

\begin{tabular}{cccccccccc}
\hline Parameter $(\mathrm{nm})$ & $a$ & $c$ & $\sigma_{1,2}{ }^{x, y}$ & $\sigma_{1,2} z$ & $\sigma_{3}{ }^{x, y}$ & $\sigma_{3}{ }^{z}$ & $R_{x, y}$ & $R_{z}$ & $\sigma_{R}$ \\
\hline QD1 & 5.1 & 4.2 & 1.6 & 0.2 & 0.7 & 0.2 & 1.6 & 1.0 & 0.4 \\
\hline
\end{tabular}


(a)

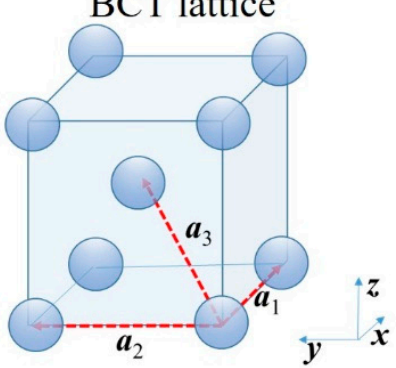

(b)

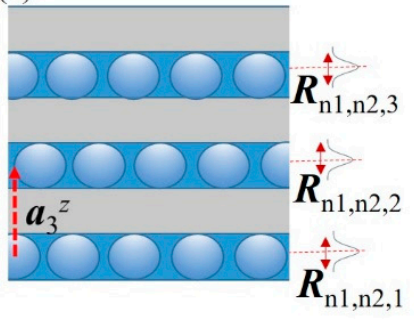

(c)

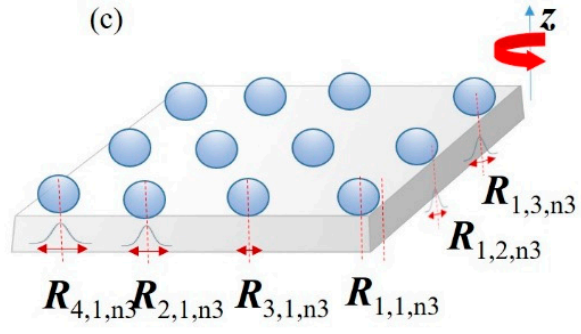

Figure 3. (a) Basis vectors $\boldsymbol{a}_{1}-\boldsymbol{a}_{3}$ of the body-centered tetragonal (BCT) QD lattice used for the GISAXS modeling. (b) Arrangement of QDs within the layers that have long-range ordering (LRO) in the $z$ direction. The positions of the QDs within each layer fluctuate around predefined $z$ positions. The position of the $n$-th QD within the layer $\boldsymbol{R}_{n 1, n 2, n 3}$ are denoted in the figure. (c) Model of the QD ordering within a single layer of the multilayer stack. The QDs have short-range ordering (SRO) within each layer due to the self-assembly mechanism of their growth, and no predefined positions.

\subsection{D Networks of Ge Nanowires}

The second example shows a 3D network of Ge nanowires grown also by the self-assembly growth presented in the previous example, but with no multilayer deposition. The deposition consisted of continuous $\mathrm{Ge}+\mathrm{Al}_{2} \mathrm{O}_{3}$ co-deposition. In Figure 4, we present GISAXS maps and their simulations for two films differing by the NW length and the parameters of the NW lattice. The parameters of the simulations are given in Table 2. Details of their preparation are given in Section 2. For this case, there are no predefined positions for the network nodes; thus, all components of the $G_{3 D}$ function are $S R O$, as shown below.

$$
G_{3 D}(\boldsymbol{q})=G_{S R O}^{(1)}(\boldsymbol{q}) G_{S R O}^{(2)}(\boldsymbol{q}) G_{S R O}^{(3)}(\boldsymbol{q})
$$
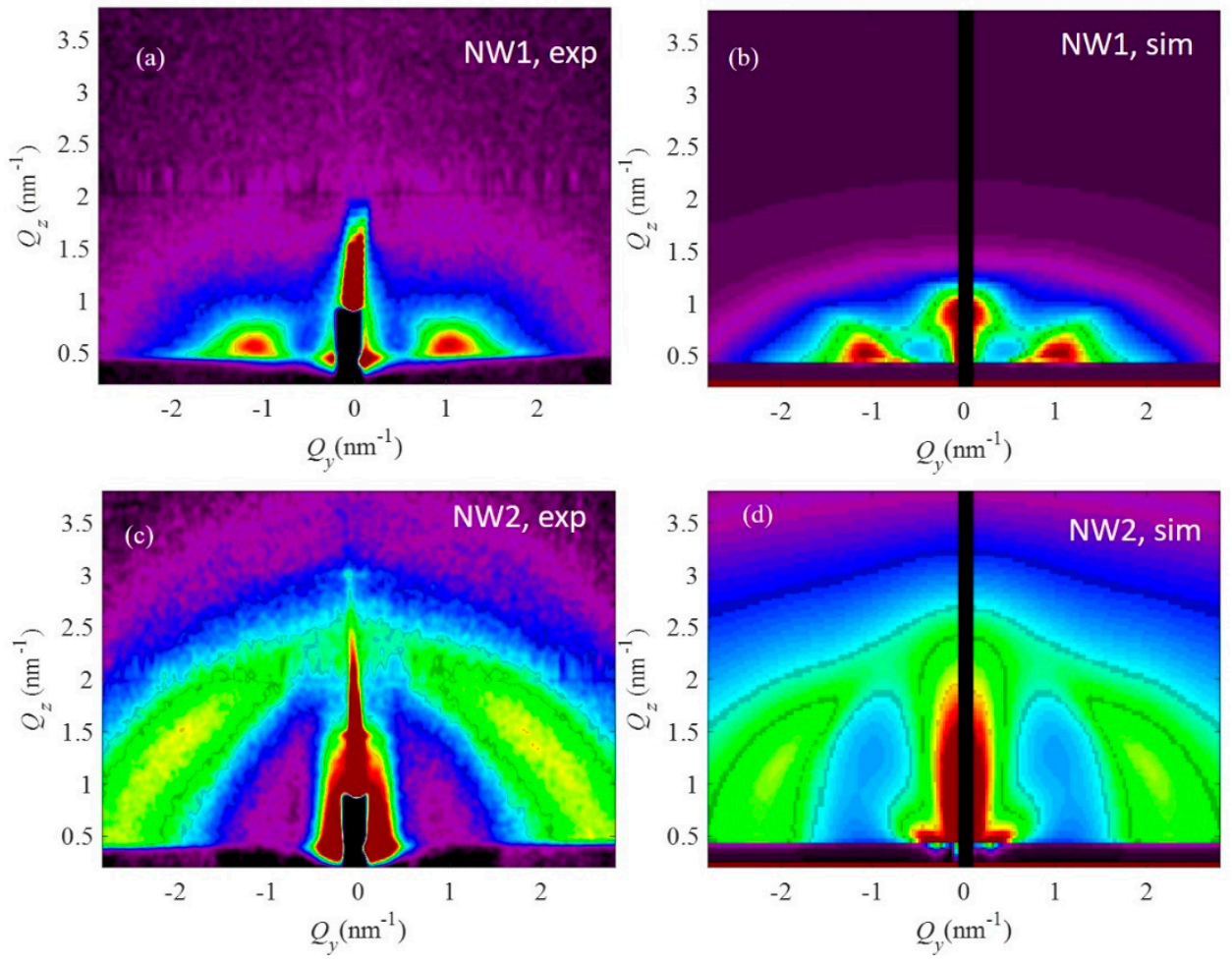

Figure 4. GISAXS maps of nanowire (NW) networks NW1 and NW2 (a,c) and their simulations $(\mathbf{b}, \mathrm{d})$, respectively. 
Table 2. Parameters of the simulation shown in Figure 4b,d. NW—nanowire.

\begin{tabular}{cccccccccc}
\hline Parameter $(\mathrm{nm})$ & $\boldsymbol{a}$ & $c$ & $\sigma_{\mathbf{1 , 2}}{ }^{x, y}$ & $\sigma_{\mathbf{1 , 2}}{ }^{z}$ & $\sigma_{\mathbf{3}}{ }^{x, y}$ & $\sigma^{z}$ & $\boldsymbol{R}_{x, y}$ & $\boldsymbol{R}_{\boldsymbol{z}}$ & $\sigma_{\boldsymbol{R}}$ \\
\hline NW1 & 5.5 & 6.3 & 1.2 & 1.8 & 0.8 & 1.6 & 1.0 & 2.3 & 0.4 \\
\hline NW2 & 2.9 & 1.9 & 0.9 & 0.9 & 1.2 & 3.7 & 0.4 & 0.8 & 0.1 \\
\hline
\end{tabular}

However, the complication in this example is the shape of the NWs. The network is continuous, and its geometry is shown in Figure 5. In an ideal case, this structure consists of cylinders connected in the nodes of a BCT lattice, as shown in Figure 5a. In the real case, there are deviations in the network node positions, as visible from the cross-sectional TEM image of this structure (Figure $5 b$ ). The form factor of this shape has a complicated form, and we prefer to have simple analytical formulas for GISAXS fitting, because we wish to have a fast fitting program. Therefore, we use an approximation choosing spheroidal QDs arranged in a BCT lattice (the same as in the previous example), but with the QDs tilted along the basis vector $\boldsymbol{a}_{3}$ as shown in the Figure 5c. $R_{x, y}$ denote the radii of the NWs, and $R_{z}$ is the half of their length. Here, we have to perform averaging over all four equivalent configurations of the basis vectors $\boldsymbol{a}_{3}$ (connection of the center of the BCT lattice to the four neighboring nodes in the $\boldsymbol{a}_{1}-\boldsymbol{a}_{2}$ plane), as well as azimuthal averaging.

(a)

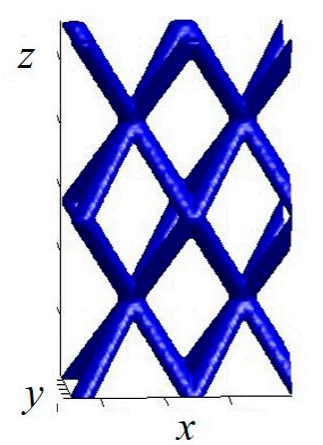

(b)

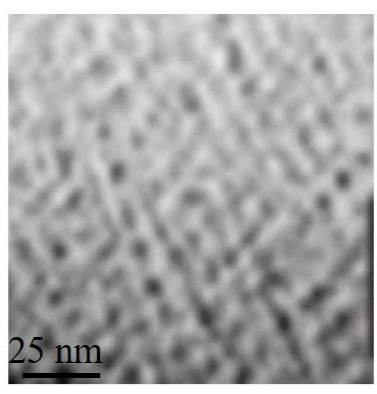

(c) BCT lattice

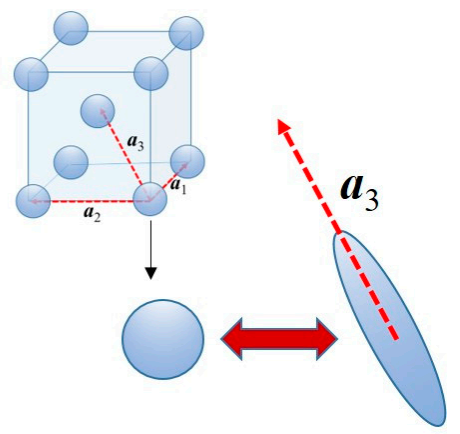

Figure 5. (a) Model of ideal three-dimensional (3D) nanowire networks. They consist of cylinders connected in the nodes of a BCT lattice. (b) Transmission electron microscopy image of the film cross-section made perpendicular to the substrate. (c) Schematic presentation of the model used in the GISAXS simulation. The QDs used in the previous example are replaced by elongated QDs tilted along the basis vector $\boldsymbol{a}_{3}$.

Using the presented model, we get a good agreement between the experimental and the simulated intensity distributions. The parameters obtained are also in good agreement with the microscopic measurements [22].

\subsection{Lattices of Core/Shell Quantum Dots}

In this example, we demonstrate how the internal structure of QDs can influence the analysis of a GISAXS map. The production process of the core/shell QDs presented in this example is very similar to that in Section 4.1, except for the fact that a very thin Si layer is deposited after each Ge layer, i.e., it is a Ge/Si/ $/ \mathrm{Al}_{2} \mathrm{O}_{3}$ multilayer deposition (for details, please see Section 2). Due to such a deposition process, each Ge QD is covered by a thin (about $1 \mathrm{~nm}$ thick) Si shell, and this shell is not symmetric around the core. This process is analyzed in detail in References [10,11]. Hence, the shape and ordering type of the QDs is the same as in Section 4.1, but the internal structure of the QDs is slightly different. It seems reasonable that a different internal structure of the QDs leads to different GISAXS intensity distributions, and no additional story about it is necessary. However, here, we want to point out that a very tiny difference in the QD structure, like a shell with the thickness of a few 
angstroms that is often invisible by microscopy, can cause very strong effects and cause problems in the GISAXS analysis (please see Reference [10] for an example). In many cases, we are not even aware that such a shell exists around NOs that we wish to investigate, because a chemical reaction easily occurs at the interface between the NO and the matrix that surrounds it to form a shell. Therefore, this example illustrates how a thin shell affects a GISAXS intensity distribution.

The correct choice of the shape factor is a Fourier transform of the core-shell nanoparticle with a shifted origin (details about the structure can be found in References $[10,11]$ ). The experimentally measured map and the simulation obtained by fitting using that shape factor are given in Figure $6 \mathrm{a}, \mathrm{b}$, respectively. However, if we apply the same model as in Section 4.1 (BCT ordering of spheroidal QDs), we can also get a reasonably good fit and good agreement between the experiment and its simulation (Figure 6c). The parameters for both simulations are given in the Table 3. We can see that the obtained sizes for the QDs, especially their vertical sizes, are completely wrong. Only after microscopy analysis of the material cross-section, shown in Figure $6 \mathrm{~d}$, the correct model was identified. The simulation with the core-shell shape factor gives a very good agreement with the microscopy measurements (Figure 6e). However, the sizes obtained by the simple model are very different from the real values (Figure 6f). With a careful inspection of the simulation Sim 2, we can notice that the low intensities (at the higher $Q_{z}$ ) are different from in the experiment. Here, we see it because of the logarithmic scale for the intensity distribution; for the linear case, it could be easily overlooked. If we use larger radius values, then we cannot get any good agreement for the peak shape and intensities. Thus, a very careful analysis is needed. The effect depends strongly on the material of the core and shell, i.e., on their electron density contrast. Generally, the strongest difference should have a stronger effect on the GISAXS intensity distribution.
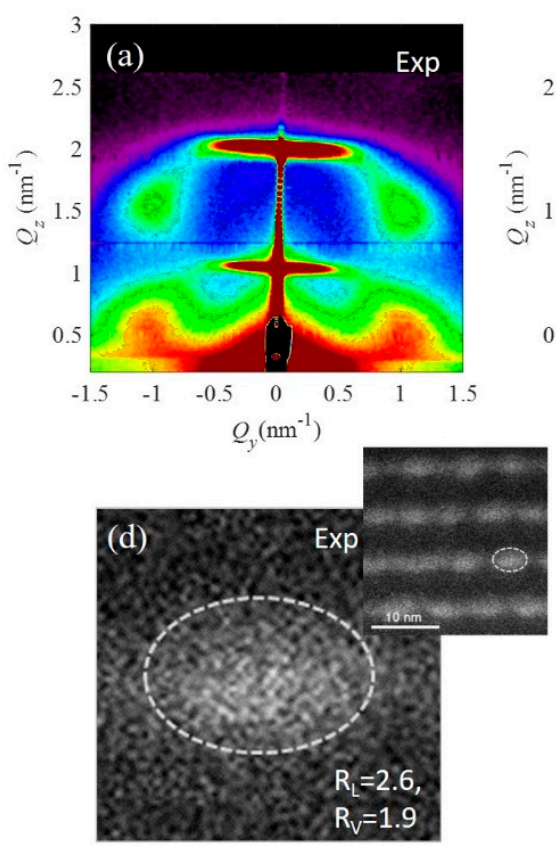

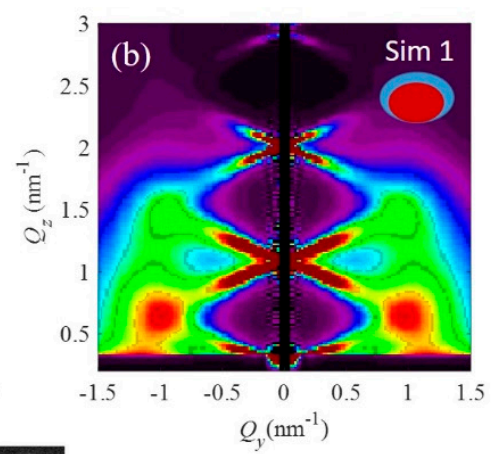

(e)

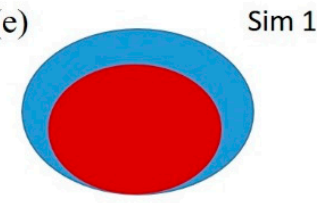

$\mathrm{R}_{\mathrm{L}}=2.6$

$\mathrm{R}_{\mathrm{V}}=2.0$

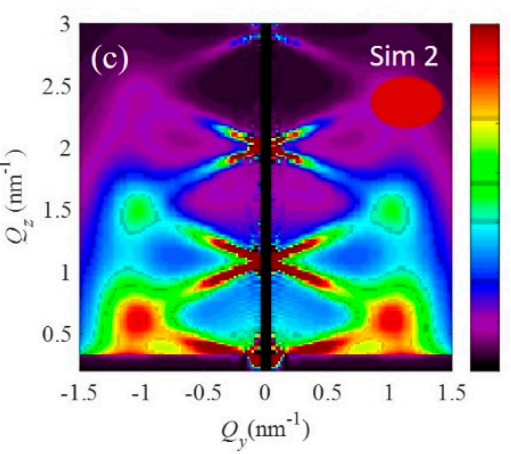

(f)

$\operatorname{Sim} 2$

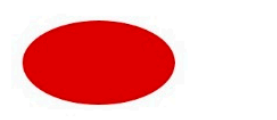

$\mathrm{R}_{\mathrm{L}}=1.8$,

$\mathrm{R}_{\mathrm{v}}=1.1$

Figure 6. (a) Experimental GISAXS map of Ge/Si core/shell quantum dots in alumina [11]. (b) Simulation of the experimental map using the shape factor for core/shell QDs with shifted origin [10]—Sim 1. (c) Simulation of the experimental map using the shape factor for spheroidal QDs-Sim 2. (d) Transmission electron microscopy image of the same film demonstrating the real size of the QDs. $R_{L}$ denotes the radius parallel to the multilayer, and $R_{\mathrm{V}}$ denotes that perpendicular to it. Model of the structure properties of QDs obtained from (e) Sim 1 and (f) Sim 2. 
Table 3. Parameters of the two simulations shown in Figure $6 b, c$ for the film GeSi1; $t$ is the shell thickness, and $d$ is the shift of the core with respect to the center of the shell.

\begin{tabular}{cccccccccccc}
\hline Parameter (nm) & $a$ & $c$ & $\sigma_{\mathbf{1 , 2}}{ }^{x, y}$ & $\sigma_{\mathbf{1 , 2}}{ }^{z}$ & $\sigma_{\mathbf{3}}{ }^{x, y}$ & $\sigma^{z}$ & $\boldsymbol{R}_{x, y}$ & $\boldsymbol{R}_{\boldsymbol{z}}$ & $\sigma_{\boldsymbol{R}}$ & $\boldsymbol{t}$ & $\boldsymbol{d}$ \\
\hline $\operatorname{Sim} 1$ & 5.2 & 6.3 & 1.5 & 0.2 & 1.4 & 0.6 & 2.6 & 2.0 & 0.3 & 0.9 & 0.3 \\
\hline $\operatorname{Sim} 2$ & 5.2 & 6.5 & 1.9 & 0.3 & 1.1 & 0.5 & 1.8 & 1.1 & 0.3 & - & - \\
\hline
\end{tabular}

\section{Discussion}

In the first example (given in Section 4.1), it was illustrated how to distinguish LRO and SRO in the particular space directions and how the ordering type influences the peak profiles. To be able to distinguish the SRO and LRO types of ordering, we should have at least two orders of peaks visible in the GISAXS maps. Of course, we can also determine it from the preparation process of the material if we know it. For example, if the entire material grows only by a self-assembly process, like for the Ge NWs given in the second example (Section 4.2), the SRO model should be applied in all directions. Often, we have a combination of different ordering types in different directions, as also shown in Section 4.1, and several nice examples showing combinations of all three ordering types (SRO, LRO, and $\mathrm{RO}$ ) are given in Reference [2]. In that paper the analysis of GISAXS maps is considered from the nanostructures produced by ion modification of material surfaces. Ordered NOs form along the trace of each ion on the material surface. These NOs have SRO along the ion track, and LRO in the direction perpendicular to it, due to the predefined positions along the ion track. If the irradiation fluence (number of used ions per surface unit) is low, then these traces have RO; however, if the fluence is high, the traces overlap and a superstructure of ion traces is formed which has SRO type.

From the third example (Section 4.3), we can conclude that a very wrong result can be obtained even if the fit results are reasonably in good agreement with the experiment. The problem is of course the shape factor. This is one of the main disadvantages of the GISAXS method: different structures can produce the same or very similar GISAXS maps, and if we do not know enough details about the material, and if we do not have complemental measurements, it is very easy to get completely wrong results.

Finally, we wish to discuss the central region of the GISAXS maps, close to the $Q_{y}=0$ axis. This region contains a contribution of the coherent scattering that is not taken into account in the used simulations. Additionally, it also contains the contribution from the interface roughness in the multilayer samples, (not taken into account in the simulations). If we take into account all these contributions, the number of fitting parameters will be huge, and the fit would be completely unreliable. Therefore, in the GISAXS analysis, we do not take this central part into consideration. In addition, the paracrystal-based models that we use for the simulations do not work well in this region because the shape effects of the entire QD lattice become significant there. Therefore, cross-like structures appear in this part in the simulations, instead of sheets (please see the GISAXS simulations in the given examples). Only the central position (at the $Q_{y}=0$ plane) of this cross-structure is correct and we should take it into account. An illustration of how to select properly the region of interest for GISAXS is shown in the Figure 7.

As mentioned in the introduction, a new computer program called GisaxStudio [19] is under development. It enables the selection of the region of interest of the desired shape for the fitting. It contains the different models mentioned here (3dLattice for 3D lattices of nanoparticles) and also in Reference [2] (iBeam for ion-beam-modified materials). We hope that it will not be useful only for researchers for whom the structural characterization of nanomaterials is the primary research interest, but also for everyone who is just "passing by". The software is also designed to grow; thus, it can be used for different types of materials in the future. The latest version is available in Reference [19], and a paper describing it precisely is in the preparation process [23]. 


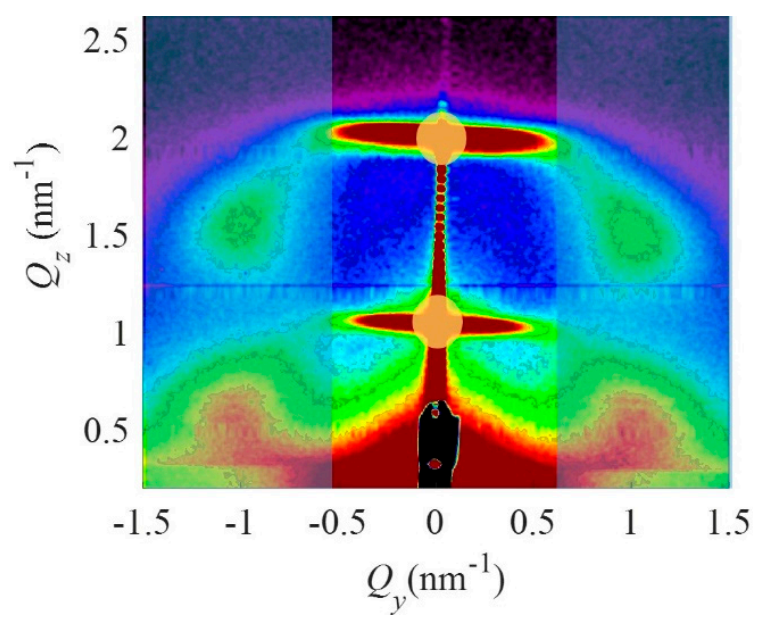

Figure 7. Example how to choose the region of interest for fitting of a GISAXS experimental map. The light-blue shaded areas should be included in the fit, while the positions of the sheets in the yellow shaded areas should be adjusted to fit the position of the crosses in the simulated maps.

\section{Conclusions}

In this paper, we showed some basic principles of GISAXS data analysis. It shows the importance of the proper choice of models for the description of the arrangement and shape of nano-objects within a matrix. The correct model type can be determined by careful analysis of the nano-object formation process, and from the experimental GISAXS maps from the materials containing nano-objects. We also pointed out some main steps in the analysis of GISAXS data. It is especially suitable for beginners in the usage of the GISAXS technique.

Author Contributions: Methodology, M.M., L.B., and K.S.; software, M.M., K.S., L.B., and I.M.; analysis, M.M., and L.B.; data curation, K.S., M.M., L.B., and S.B.; writing—original draft preparation, L.B., and M.M.; review and editing, all authors.

Funding: This research was funded by the Croatian Science Foundation (pr. no IP-2018-01-3633), "Center of Excellence for Advanced Materials and Sensing Devices" (Grant No. KK.01.1.1.01.0001), and CalipsoPlus project (pr. no 20182085). The authors are grateful to J. Erceg for help with the sample preparation and D. Mičetic for the GISAXS measurements.

Conflicts of Interest: The authors declare no conflicts of interest.

\section{References}

1. Renaud, J.; Lazzari, R.; Leroy, F. Probing surface and interface morphology with Grazing Incidence Small Angle X-Ray Scattering. Surf. Sci. Rep. 2009, 64, 255-380. [CrossRef]

2. Buljan, M.; Karlušić, M.; Nekić, N.; Jerčinović, M.; Bogdanović-Radović, I.; Bernstorff, S.; Radić, N.; Mekterović, I. GISAXS analysis of ion beam modified films and surfaces. Comp. Phys. Commun. 2017, 212, 69-81. [CrossRef]

3. Buljan, M.; Radić, N.; Bernstorff, S.; Dražić, G.; Bogdanović-Radović, I.; Holý, V. Grazing incidence small angle x-ray scattering: Application to the study of quantum dot lattices. Acta Cryst. A 2012, 68, 124-138. [CrossRef] [PubMed]

4. Buljan, M.; Desnica, U.V.; Ivanda, M.; Radić, N.; Dubček, P.; Dražić, G.; Salamon, K.; Bernstorff, S.; Holý, V. Formation of three-dimensional quantum-dot superlattices in amorphous systems: Experiments and Monte Carlo simulations. Phys. Rev. B 2009, 79, 035310. [CrossRef]

5. Buljan, M.; Pinto, S.R.C.; Rolo, A.G.; Martín-Sánchez, J.; Gomes, M.J.M.; Grenzer, J.; Mücklich, A.; Bernstorff, S.; Holý, V. Self-Assembling of Ge quantum dots in an alumina matrix. Phys. Rev. B 2010, 82, 235407. [CrossRef]

6. Buljan, M.; Jerčinović, M.; Siketić, Z.; Bogdanović-Radović, I.; Delač Marion, I.; Kralj, M.; Ivanda, M.; Turković, A.; Dražić, G.; Bernstorff, S.; et al. Tuning the growth properties of Ge quantum dot lattices in amorphous oxides by matrix type. J. Appl. Cryst. 2013, 46, 1490-1500. [CrossRef] 
7. Buljan, M.; Roshchupkina, O.; Šantić, A.; Holý, V.; Baehtz, C.; Mücklich, A.; Horák, L.; Valeš, V.; Radić, N.; Bernstorff, S.; et al. Growth of a three-dimensional anisotropic lattice of Ge quantum dots in an amorphous alumina matrix. J. Appl. Cryst. 2013, 46, 709-715. [CrossRef]

8. Buljan, M.; Radić, N.; Ivanda, M.; Bogdanović-Radović, I.; Karlušić, M.; Grenzer, J.; Prucnal, S.; Dražić, G.; Pletikapić, G.; Svetličić, V.; et al. Ge quantum dot lattices in $\mathrm{Al}_{2} \mathrm{O}_{3}$ multilayers. J. Nanopart Res. 2013, 15, 1485. [CrossRef]

9. Jerčinović, M.; Radić, N.; Buljan, M.; Grenzer, J.; Delač-Marion, I.; Kralj, M.; Bogdanović-Radović, I.; Hübner, R.; Dubček, P.; Salamon, K.; et al. Self-Assembled growth of Ni nanoparticles in amorphous alumina matrix. J. Nanopart Res. 2014, 16, 2296. [CrossRef]

10. Buljan, M.; Radić, N.; Sancho-Paramon, J.; Janicki, V.; Grenzer, J.; Bogdanović-Radović, I.; Siketić, Z.; Ivanda, M.; Utrobičić, A.; Hübner, R.; et al. Production of three-dimensional quantum dot lattice of Ge/Si core-shell quantum dots and Si/Ge layers in an alumina glass matrix. Nanotechnology 2015, 26, 065602. [CrossRef] [PubMed]

11. Nekić, N.; Sancho Parramon, J.; Bogdanović-Radović, I.; Grenzer, J.; Huebner, R.; Ivanda, M.; Buljan, M. Ge/Si core/shell quantum dots in alumina: Tuning the optical absorption by the core and shell size. Nanophotonics 2017, 6, 1055-1062. [CrossRef]

12. Nekić, N.; Šarić, I.; Salamon, K.; Basioli, L.; Sancho-Parramon, J.; Grenzer, J.; Hübner, R.; Bernstorff, S.; Petravić, M.; Mičetić, M. Preparation of non-oxidized Ge quantum dot lattices in amorphous $\mathrm{Al}_{2} \mathrm{O}_{3}, \mathrm{Si}_{3} \mathrm{~N}_{4}$ and SiC matrices. Nanotechnology 2019, 30, 335601. [CrossRef] [PubMed]

13. Sinha, S.K.; Sirota, E.B.; Garoff, S.; Stanley, H.B. X-ray and neutron scattering from rough surfaces. Phys. Rev. $B$ 1988, 38, 2297-2311. [CrossRef] [PubMed]

14. Lazzari, R. IsGISAXS: A program for grazing-incidence small-angle X-ray scattering analysis of supported islands. J. Appl. Cryst. 2002, 35, 406-421. [CrossRef]

15. Tate, M.P.; Urade, V.N.; Kowalski, J.D.; Wei, T.C.; Hamilton, B.D.; Eggiman, B.W.; Hillhouse, H.W. Simulation and interpretation of 2D diffraction patterns from self-assembled nanostructured films at arbitrary angles of incidence: From grazing incidence (above the critical angle) to transmission perpendicular to the substrate. J. Phys. Chem. B 2006, 110, 9882-9892. [CrossRef] [PubMed]

16. Babonneau, D. FitGISAXS: Software package for modelling and analysis of GISAXS data using IGOR Pro. J. Appl. Cryst. 2010, 43, 929-936. [CrossRef]

17. Chourou, S.; Sarje, A.; Li, X.S.; Chan, E.; Hexemer, A. HipGISAXS: A High Performance Computing Code for Simulating Grazing Incidence X-Ray Scattering Data. J. Appl. Crystallogr. 2013, 46, 1781-1795. [CrossRef]

18. Durniak, C.; Ganeva, M.; Pospelov, G.; Van Herck, W.; Wuttke, J. BornAgain—Software for Simulating and Fitting X-ray and Neutron Small-Angle Scattering at Grazing Incidence 2015. Available online: http://juser.fz-juelich.de/record/255761 (accessed on 11 September 2019).

19. Mekterović, I.; Basioli, L.; Mičetić, M. GISAXStudio 2019. Available online: http://homer.zpr.fer.hr/gisaxstudio/ doku.php?id=install (accessed on 11 September 2019).

20. Ragulskaya, A.V.; Andreeva, M.A.; Rogachev, A.V.; Yakunin, S.N. The investigation of [Fe/Cr] multilayer by GISAXS. Superlattices Microstruct. 2019, 125, 16-25. [CrossRef]

21. Eads, J.L.; Millane, R.P. Diffraction by the ideal paracrystal. Acta Cryst. A 2001, 57, 507-517. [CrossRef] [PubMed]

22. Ray, N.; Gupta, N.; Adhikary, M.; Nekić, N.; Basioli, L.; Dražić, G.; Bernstorff, S.; Mičetić, M. Influence of Structure on Electronic Charge Transport in 3D Ge Nanowire Networks in an Alumina Matrix. Sci. Rep. 2019, 9, 5432. [CrossRef] [PubMed]

23. Mekterović, I.; Basioli, L.; Bernstorff, S.; Mičetić, M. GisaxStudio—Software platform for GISAXS analysis of nanostructured materials. Unpublished work. 2019.

(C) 2019 by the authors. Licensee MDPI, Basel, Switzerland. This article is an open access article distributed under the terms and conditions of the Creative Commons Attribution (CC BY) license (http://creativecommons.org/licenses/by/4.0/). 